\title{
Novas funções para estimar a produção de leite, em 305 dias de lactação, de vacas da raça Gir ${ }^{1}$
}

\author{
Geovanne Ferreira Rebouças ${ }^{2}$, Tarcisio de Moraes Gonçalves ${ }^{3}$, Mário Luiz Martinez ${ }^{4}$, Jairo \\ Azevedo Junior ${ }^{5}$, Wiebe Koops ${ }^{6}$
}

\author{
1 Projeto financiado pela FAPEMIG e pelo CNPq. \\ 2 Programa de Pós-graduação em Zootecnia - UFLA - Lavras, MG. \\ ${ }^{3}$ Departamento de Zootecnia da UFLA. \\ ${ }^{4}$ Embrapa-CNPGL. \\ ${ }^{5}$ Curso de graduação em Zootecnia - UFLA \\ ${ }^{6}$ Universidade de Wageningen - Holanda.
}

RESUMO - Os objetivos neste estudo foram, a partir da forma generalizada da função de Michaelis-Menten, derivar novas funções, acumulada e diária, para estimar a produção de leite, em 305 dias de lactação, de vacas da raça Gir, usando informações de produção de leite no dia do controle leiteiro. Foram utilizadas 7.412 lactações de 3.416 vacas da raça Gir com partos entre 1987 e 2004, distribuídos em 51 rebanhos participantes do Programa Nacional de Melhoramento Genético do Gir Leiteiro, coordenado pela Embrapa Gado de Leite e pela Associação Brasileira dos Criadores de Gir Leiteiro. Foram ajustados os modelos para as subclasses nível de produção (Alto $\geq 2.628$ kg de leite na lactação; Baixo <2.628 kg de leite na lactação), estação de parição (Seca e Águas) e ordem de parição (OP 1 a 5 e >5). Os valores do desvio-padrão residual variaram de 0,131 a 0,984, os do Coeficiente de Determinação $\left(\mathrm{R}^{2}\right)$ foram próximos de 1 e de Durbin-Watsom foram próximos de 2. A estatística de Theil (U) apresentou valores de $\mathrm{U}^{\mathrm{M}}$ e $\mathrm{U}^{\mathrm{V}}$ próximos de 0 e de $\mathrm{U}^{\mathrm{C}}$ próximos de 1 , sugerindo acurácia das estimativas. Estes resultados indicam bom ajuste da função. A persistência da lactação relaciona-se ao parâmetro d e o tempo de ocorrência do pico da lactação se relaciona simultaneamente com os parâmetros d e n. Então, as novas funções ajustam-se bem a lactações de vacas da raça Gir e são caracterizadas pela simplicidade no procedimento de ajuste, pela facilidade de interpretação biológica dos parâmetros e pela flexibilidade, ou seja, é possível obter a função acumulada a partir da função diária. As novas funções apresentam-se como funções alternativas para projetar lactações não encerradas e podem ser utilizadas na avaliação genética de animais em programas de melhoramento genético.

Palavras-chave: curva de lactação, produção de leite acumulada, produção de leite diária, vacas da raça Gir

\section{New functions to estimate 305-days milk production of Gir cows}

\begin{abstract}
This study aimed to calculate new accumulated and daily functions based on the Michaelis-Menten equation to estimate the 305-days production of Gir cows using test day milk yields. Data consisted of 7,412 lactation records of 3,416 Gir cows (Bos indicus) collected from 1987 to 2004 in 51 herds assisted by the National Breeding Program of the Dairy Gir, coordinated by the Dairy Cattle National Research Center (EMBRAPA) and the Brazilian Association of Dairy Gir Breeders (ABCGIL). The models included the effects of herd milk production (High and Low), calving season (dry e rainy) and parity order (OP 1 to 5 and $>5$ ). Residual Standard Deviation values ranged from 0.131 to 0.z984. Estimates for the Coefficient of Determination $\left(\mathrm{R}^{2}\right)$ were close to one and for the Durbin-Watson coefficient were close to two. Values of statistics of Theil (U) were close to zero for $\mathrm{U}^{\mathrm{M}}$ and $\mathrm{U}^{\mathrm{V}}$ and close to one for $\mathrm{U}^{\mathrm{C}}$ suggesting a good fitting for the function. The persistency of milk yield is related to the parameter $d$ and the time of the pick yield is related to both parameters $d$ and n. The new functions are characterized by simplicity of the fitting procedure, biological interpretability of the parameters and flexibility to convert the daily function to the accumulated function. The new functions are an alternative to project lactations with records in progress to estimate the production required in genetic evaluation systems based on 305-day milk production records.
\end{abstract}

Key Words: cumulative milk yield, daily milk yield, Gir cows, lactation curve

\section{Introdução}

Atualmente, na moderna exploração leiteira, um rígido controle da produção é fator determinante para o sucesso da atividade. Uma forma prática e consistente de se obter este controle é por meio do estudo da curva de lactação dos animais.

A curva de lactação é caracterizada pela produção de leite ao longo da lactação e pode ser dividida em três fases. 
Produções iniciais, pico de produção e taxa de decréscimo após o pico, definida como persistência da lactação (Pineda, 1987).

Vacas zebuínas ou mestiças tendem a apresentar curvas de lactação iniciando no pico de produção, ou sem a fase de inclinação do parto ao pico (Papajcsik \& Bodero, 1988).

É necessário o conhecimento de outras características da curva de lactação, além do pico, como a persistência da lactação, pois, a partir do pico, a produção tende a cair em uma taxa mais ou menos constante até o fim da lactação, que coincide com o período em que a vaca prepara-se para uma nova lactação (Keown \& Van Vleck, 1972).

O conhecimento da curva de lactação de um animal pode ajudar o pecuarista na adequação de técnicas de alimentação e manejo. Outra aplicação do estudo de curvas de lactação é na estimação da produção total de leite, a partir de registros incompletos, o que torna possível detectar quando um rebanho ou uma vaca não apresenta o desempenho esperado (Morant \& Gnanasakthy, 1989).

Existem atualmente muitas funções que estimam a produção leiteira diária dos animais, porém, nenhuma função apresentada até o momento estima a produção de leite acumulada dos animais ainda em lactação.

Em muitos estudos de comparação de modelos para estimar a produção de leite é preciso estimar a produção de leite total durante um tempo de lactação fixo, principalmente aos 305 dias. Também para propósitos de seleção é importante ser capaz de estimar a produção de leite aos 305 dias em uma fase inicial da lactação. Embora haja várias funções para descrever a curva de lactação diária, como a função de Wood ou Gamma incompleta (Wood, 1967), as funções multifásicas (Koops et al., 1987), a função de Wilmink (1987) e a função de Shaeffer \& Dekkers (1994), entre outras. A maioria destas funções não tem integral conhecida necessária para o estudo de produções acumuladas. Assim, para que se possam fazer estudos da produção acumulada, torna-se necessária a derivação de funções específicas.

A importância dessa função pode ser caracterizada, principalmente pela obtenção imediata da produção de leite acumulada em tempo determinado da lactação, não sendo necessária a multiplicação da produção média pelo número de dias transcorridos da lactação.

Os objetivos neste estudo foram derivar simultaneamente novas funções, acumulada e diária, para estimar a produção de leite em 305 dias de lactação de vacas da raça Gir usando informações de produção de leite no dia do controle leiteiro.

\section{Material e Métodos}

Foram utilizados dados de 7.412 lactações de 3.416 vacas da raça Gir, cujos partos ocorreram no período de 1987 a 2004, distribuídos em 51 rebanhos da raça Gir explorados para produção de leite e participantes do Programa Nacional de Melhoramento Genético do Gir Leiteiro, coordenado pela Embrapa Gado de Leite (CNPGL) e pela Associação Brasileira dos Criadores de Gir Leiteiro (ABCGIL).

A preparação dos dados foi realizada no Departamento de Zootecnia (DZO) da Universidade Federal de Lavras (UFLA) utilizando-se o programa Statistical Analysis System (SAS) (1996).

Após análise de variância, todas as produções no dia do controle leiteiro foram corrigidas simultaneamente para os efeitos não-genéticos de: ano de parição (1987 a 2004), estação de parição, rebanho, ordem de parição e número do controle leiteiro. O efeito não-genético de rebanho foi classificado de acordo com as médias de produção de leite em 305 dias para vacas que pariram com até 60 meses de idade, em duas classes de produção: baixa (rebanhos com produção de leite em 305 dias menor que $2.628 \mathrm{~kg}$ ) e alta (rebanhos com produção de leite em 305 dias maior ou igual a $2.628 \mathrm{~kg}$ ).

Duas classes de estações de parição foram consideradas: estação da seca (meses de parição de abril a setembro) e estação das águas (meses de parição de outubro a março) e foram adotadas as seguintes classes de ordem de parição: 1 a $5(\mathrm{OP}=1$ a 5$)$ e maiores que $5(\mathrm{OP}>5)$.

As médias de produção de leite no dia dos 11 controles leiteiros considerados foram pré-ajustadas pelo método dos mínimos quadrados (média de mínimo quadrados), de acordo com as subclasses nível de produção, estação de parição e ordem de parição e as possíveis interações entre estes fatores de variação.

Para o estudo das curvas de lactação acumulada e diária, foi usada a função generalizada de Michaelis Menten (Neal \& Thornley, 1983):

$$
f(y \mid t)=\frac{a}{1+(d / t)^{n}}+\varepsilon
$$

em que $\mathrm{f}(\mathrm{y} \mid \mathrm{t})$ = produção de leite $\mathrm{y}$ acumulada no dia $\mathrm{t}$ da lactação; $a=$ produção estimada quando t é infinito; $\mathrm{d}=$ tempo $\mathrm{t}$ em que a produção de leite e 0,5 de a e $\mathrm{n}$ é o parâmetro relativo à inflexão (declividade) da função.

Para obter uma estimativa direta $\left(\mathrm{Y}_{305}\right)$ da produção em 305 dias, o parâmetro a foi substituído por Y $_{05}$, resultando na nova função: 


$$
\mathrm{Y}_{\mathrm{t}}=\mathrm{Y}_{305} \frac{1+(\mathrm{d} / 305)^{\mathrm{n}}}{1+(\mathrm{d} / \mathrm{t})^{\mathrm{n}}}
$$

A função [2] representa a quantidade de leite produzida no dia $\mathbf{t}$, que é a produção acumulada no período de0 até o dia t. Entretanto, na prática, a produção de leite de vacas é habitualmente medida em $\mathrm{kg}$ de leite por dia a intervalos de 2, 3 ou 4 semanas durante a lactação (controle leiteiro). Assim, derivando-se a função [2], pode-se chegar a uma nova função que descreve a produção de leite diária:

Assim, a primeira derivada da Equação [2] emt pode ser usada para descrever a produção diária de leite:

$$
\mathrm{y}_{\mathrm{t}}=\mathrm{Y}_{305} \frac{\mathrm{n}}{\mathrm{t}} \frac{1+(\mathrm{d} / 305)^{\mathrm{n}}}{\left.1+(\mathrm{d} / \mathrm{t})^{\mathrm{n}}\right]\left[1+(\mathrm{t} / \mathrm{d})^{\mathrm{n}}\right]} .
$$

A Equação [3] é uma nova função para descrever uma curva de lactação e pode ser usada para estimar a produção de leite em 305 dias de lactação diretamente a partir de dados de produção diária de leite.

Os pontos críticos da função [3], produção de leite no pico $\left(\mathrm{Y}_{\mathrm{m}}\right)$ e o tempo de ocorrência do pico $\left(\mathrm{t}_{\mathrm{m}}\right)$ foram obtidos por:

Produção no pico $\left(\mathrm{Y}_{\mathrm{m}}\right)$ :

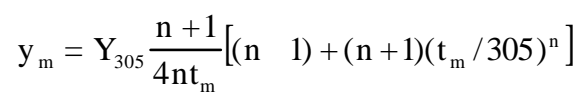

Tempo em que ocorre o pico $\left(\mathrm{t}_{\mathrm{m}}\right)$ :

$\mathrm{t}_{\mathrm{m}}=\mathrm{d}\left[\left(\begin{array}{ll}\mathrm{n} & 1\end{array}\right) /(\mathrm{n}+1)\right]^{1 / \mathrm{n}}$

Os parâmetros dos modelos foram estimados por meio de regressões não-lineares utilizando-se o método de GaussNewton, disponível no PROC NLIN (SAS, 1996).

Foram utilizados para verificar a qualidade de ajuste das funções os testes estatísticos de Durbin-Watson (DW) o desvio-padrão residual (DPR), o coeficiente de determinação $\left(\mathrm{R}^{2}\right)$, o quadrado médio do erro de estimação (QMEE) e as estatísticas de Theil ( $\mathrm{U}^{\mathrm{M}}, \mathrm{U}^{\mathrm{V}}$ e $\left.\mathrm{U}^{\mathrm{C}}\right)$. As estatísticas $\mathrm{U}^{\mathrm{M}}$, $\mathrm{U}^{\mathrm{V}}$ e $\mathrm{U}^{\mathrm{C}}$, conhecidas como as proporções das desigualdades, são usadas para medir três diferentes aspectos da qualidade de ajuste das funções estudadas. $\mathrm{U}^{\mathrm{M}}$ é a medida da proporção do viés, isto é, a extensão pela qual os valores médios dos valores preditos desviam dos valores observados, erro de tendência central. $U^{\mathrm{V}}$ é conhecida como a proporção da variância, erro atribuído às desigualdades das variâncias. Esta proporção de variância determina a habilidade da função em replicar a mesma variabilidade nos valores preditos como ocorre nos valores observados. Como $\mathrm{U}^{\mathrm{M}}$, quanto menor o valor de $\mathrm{U}^{\mathrm{V}}$, maior a habilidade da função em predizer acuradamente. A terceira estatística, $\mathrm{UC}^{\mathrm{C}}$, é conhecida como a proporção decorrente da covariância, erro atribuído à co-variância incompleta. Ela representa o erro de predição remanescente após o ajuste do modelo, ou após ter sido considerado os desvios dos valores médios. Assim, $\mathrm{U}^{\mathrm{M}}+\mathrm{U}^{\mathrm{V}}+\mathrm{U}^{\mathrm{C}}$ é igual a 1. Entretanto, os valores ideais para as três estatísticas são $\mathrm{U}^{\mathrm{M}}=\mathrm{U}^{\mathrm{V}}=0$ e $\mathrm{UC}^{\mathrm{C}}=1$.

\section{Resultados e Discussão}

Ao ajustar a nova função para descrever a curva de lactação diária dos animais, os valores dos desvio-padrões residuais (DPR) variaram de 0,131 a 0,984 (Tabela 1), o que sugere bom ajuste da função.

Os resíduos (Figura 1), independentemente do nível de produção, da estação de parição e da ordem de parição, foram simétricos, próximos de zero, e variaram de -1 a 1 , sugerindo também bom ajuste da função.

Os valores obtidos da estatística de Durbin-Watsom (DW) foram próximos de 2 (Tabela 1). Independentemente do nível de produção, da estação de parição e da ordem de parição, não houve autocorrelação residual de primeira ordem, com exceção apenas da ordem de parição $2(\mathrm{OP}=2)$ de vacas de nível de produção alto $(\mathrm{NIV}=1)$ paridas na estação das águas (EST =2), uma vez que o resultado para o teste DW foi significativo $(\mathrm{P}<0,001)$, indicando autocorrelação de primeira ordem. Os resultados de DW indicam bom grau de ajustamento da função, que, em 95,83\% das classes ajustadas, não apresentou autocorrelação residual de primeira ordem.

Os coeficientes de determinação $\left(\mathrm{R}^{2}\right)$ variaram de 0,438 a $0,997 \mathrm{e}$, na maior parte das classes estudadas, foram maiores que 0,92 , com exceção apenas para OP $>5$ de vacas de NIV = 1 na estação de parição seca $(E S T=1)$, que apresentou $\mathrm{R}^{2}$ de 0,438 (Tabela 1 ). Os valores de $\mathrm{R}^{2}$ foram altos e próximos de 1, o que também comprova o bom ajustamento da função para descrever uma lactação quando se ajusta a regressão das produções no dia do controle leiteiro de acordo com os estágios da lactação.

A avaliação do quadrado médio do erro de estimação (QMEE) quando ajustada a nova função foi realizada utilizando-se a estatística de Theil. Os valores de $\mathrm{UM}^{\mathrm{M}} \mathrm{UV}^{\mathrm{V}}$ foram baixos e próximos de zero $(<0,016729)$ e os valores de $\mathrm{U}^{\mathrm{C}}$ foram altos e próximos de $1(>0,9829)$. Assim, os resultados desta estatística (Tabela 1) sugerem acurácia na estimação dos valores de produção de leite no dia do controle leiteiro, o que reforça o bom ajuste da nova função.

Observando os valores estimados dos parâmetros na Tabela 1 e também nas Figuras 2 e 3, constata-se que a nova 
Tabela 1 - Número de lactações (N), produção de leite aos 305 dias de lactação $\left(\mathrm{Y}_{305}\right)$, parâmetro d e $\mathrm{n}$, teste de Durbin-Watson (DW), desvio-padrão residual (DPR), coeficiente de determinação $\left(R^{2}\right)$, quadrado médio do erro de estimação (QMEE) e estatística de Theil $\left(U^{M}, U^{V}\right.$ e $\left.U^{C}\right)$ em vacas da raça Gir provenientes de rebanhos com níveis de produção alto e baixo paridas na estação seca e das águas com ordens de parição (OP) de 1 a 5 e maiores que $5(>5)$

\begin{tabular}{|c|c|c|c|c|c|c|c|c|c|c|c|}
\hline $\mathrm{OP}$ & $\mathrm{N}$ & $\mathrm{Y}_{305}$ & d & $\mathrm{n}$ & DW & DPR & $\mathrm{R}^{2}$ & QMEE & $\mathrm{U}^{\mathrm{M}}$ & $\mathrm{U}^{\mathrm{V}}$ & $\mathrm{U}^{\mathrm{C}}$ \\
\hline & \multicolumn{11}{|c|}{ Nível de produção alto } \\
\hline & \multicolumn{11}{|c|}{ Seca (3) } \\
\hline 1 & 752 & $3.085,05$ & 513,802 & 1,190 & 1,983 & 0,155 & 0,989 & 0,0167 & 0,000121 & 0,000868 & 0,9990 \\
\hline 2 & 462 & 3.566 .79 & 461,399 & 1,135 & 2,003 & 0,162 & 0,996 & 0,0190 & 0,000030 & 0,000011 & 0,9999 \\
\hline 3 & 250 & $3.753,58$ & 445,225 & 1,110 & 1,522 & 0,274 & 0,991 & 0,0347 & 0,000117 & 0,007707 & 0,9922 \\
\hline 4 & 147 & $4.194,67$ & 438,617 & 1,121 & 2,540 & 0,290 & 0,992 & 0,0613 & 0,000024 & 0,000374 & 0,9996 \\
\hline 5 & 74 & $4.319,31$ & 426,486 & 1,128 & 2,278 & 0,424 & 0,984 & 0,1308 & 0,000278 & 0,000195 & 0,9995 \\
\hline \multirow[t]{2}{*}{$>5$} & 43 & $3.760,98$ & 337,611 & 1,161 & 1,231 & 0,984 & 0,438 & 0,1396 & 0,000431 & 0,016729 & 0,9828 \\
\hline & \multicolumn{11}{|c|}{ Águas } \\
\hline 1 & 589 & $3.212,76$ & 859,630 & 1,077 & 1,175 & 0,383 & 0,925 & 0,1067 & 0,000005 & 0,004661 & 0,9953 \\
\hline 2 & 382 & $3.622,09$ & 427,267 & 1,137 & 0,728 & 0,349 & 0,984 & 0,0887 & 0,001634 & 0,001634 & 0,9979 \\
\hline 3 & 196 & $3.837,48$ & 401,110 & 1,124 & 1,079 & 0,285 & 0,992 & 0,0590 & 0,000926 & 0,000926 & 0,9987 \\
\hline 4 & 108 & $3.928,80$ & 300,283 & 1,213 & 1,571 & 0,417 & 0,987 & 0,1267 & 0,000140 & 0,000140 & 0,9995 \\
\hline 5 & 61 & $4.246,53$ & 440,095 & 1,101 & 0,864 & 0,805 & 0,945 & 0,4714 & 0,000688 & 0,000688 & 0,9989 \\
\hline \multirow[t]{3}{*}{$>5$} & 45 & $4.249,28$ & 386,579 & 1,146 & 2,629 & 0,533 & 0,977 & 0,2067 & 0,010052 & 0,010052 & 0,9899 \\
\hline & \multicolumn{11}{|c|}{ Nível de produção baixo } \\
\hline & \multicolumn{11}{|c|}{ Seca } \\
\hline 1 & 989 & $2.185,95$ & $1.036,257$ & 1,025 & 1,423 & 0,169 & 0,972 & 0,0208 & 0,000027 & 0,001423 & 0,9985 \\
\hline 2 & 600 & $2.691,32$ & 685,094 & 1,032 & 1,399 & 0,198 & 0,987 & 0,0286 & 0,000032 & 0,000473 & 0,9994 \\
\hline 3 & 341 & $2.875,69$ & 577,435 & 1,036 & 2,146 & 0,131 & 0,996 & 0,0125 & 0,000019 & 0,000066 & 0,9999 \\
\hline 4 & 209 & $2.880,80$ & 508,785 & 1,054 & 2,226 & 0,131 & 0,997 & 0,0125 & 0,000090 & 0,000193 & 0,9997 \\
\hline 5 & 128 & $2.877,84$ & 562,004 & 1,037 & 1,436 & 0,250 & 0,987 & 0,0454 & 0,000211 & 0,000139 & 0,9941 \\
\hline \multirow[t]{2}{*}{$>5$} & 99 & $2.836,25$ & 471,406 & 1,042 & 1,470 & 0,254 & 0,990 & 0,0471 & 0,000069 & 0,005853 & 0,9940 \\
\hline & \multicolumn{11}{|c|}{ Águas } \\
\hline 1 & 1.034 & $2.182,85$ & $1.737,400$ & 0,954 & 0,990 & 0,291 & 0,927 & 0,0471 & 0,000069 & 0,005853 & 0,9941 \\
\hline 2 & 387 & $2.720,36$ & 870,529 & 0,965 & 0,981 & 0,337 & 0,970 & 0,0614 & 0,000016 & 0,009100 & 0,9909 \\
\hline 3 & 212 & $2.882,49$ & 746,228 & 0,977 & 1,212 & 0,306 & 0,981 & 0,0829 & 0,000124 & 0,000692 & 0,9992 \\
\hline 4 & 132 & $2.978,51$ & 596,098 & 0,996 & 1,159 & 0,289 & 0,987 & 0,0601 & 0,000249 & 0,000101 & 0,9997 \\
\hline 5 & 89 & $3.046,61$ & 620,640 & 0,989 & 0,997 & 0,461 & 0,967 & 0,1548 & 0,000264 & 0,000289 & 0,9995 \\
\hline$>5$ & 83 & $2.802,37$ & 475,444 & 0,991 & 1,246 & 0,471 & 0,973 & 0,1612 & 0,000606 & 0,000027 & 0,9994 \\
\hline
\end{tabular}

função utilizada apresenta boa interpretabilidade biológica dos parâmetros.

O parâmetro d está relacionado à persistência da lactação e, quanto maiores os valores de $d$, menos acentuada a redução da produção após o pico da lactação, o que evidencia maior persistência da lactação (Figuras 2 e 3 ). Na OP = 1 , independentemente do nível de produção e da estação de parição, os maiores valores foram estimados para o parâmetro d, ordem de parição que apresentou maior persistência (Figura 2 e 3). Verificou-se ainda tendência de diminuição do valor correspondente ao parâmetro d à medida que as ordens de parição aumentaram (Tabela 1) (Figuras 2 e 3). À medida que as ordens de parição aumentaram, as lactações foram menos persistentes, confirmando o relacionamento dos menores valores do parâmetro d com menor persistência da lactação. Esse parâmetro também está relacionado ao tempo de ocorrência do pico de produção de leite na lactação $\left(\mathrm{t}_{\mathrm{m}}\right)$. Quanto menor o valor de d, maior o tempo para a lactação para atingir o pico de produção (Tabelas 1 e 2; Figuras 2 e 3).

$\mathrm{O}$ parâmetro $\mathrm{n}$ é inversamente proporcional ao parâmetro d, logo, também está relacionado à existência de pico de produção de leite na lactação e também a quantos dias, a partir do início da lactação, é observado o pico. Esta afirmação pode ser confirmada pelo valor do parâmetro $n$ (Tabela 1) e pelo parâmetro $\mathrm{t}_{\mathrm{m}}$ (Tabela 2). Quanto maior o valor do parâmetro n, mais tempo transcorre até o aparecimento do pico de produção. Quando o valor de n é igual ou menor a 1 , o animal inicia a lactação com produção máxima (Tabela 2; Figuras 2 e 3), portanto, as curvas de lactação de vacas de NIV = 2, na EST = 2 não apresentam tempo de ascensão ao pico.

Os parâmetros estimados $\left(\mathrm{Y}_{305}\right.$, d e $\left.\mathrm{n}\right)$ das funções (Tabela 1) também podem ser interpretados biologicamente por meio das curvas de lactação acumulada (Figura 4). Observando os valores estimados do parâmetro d, nota-se 
Nível de produção alto
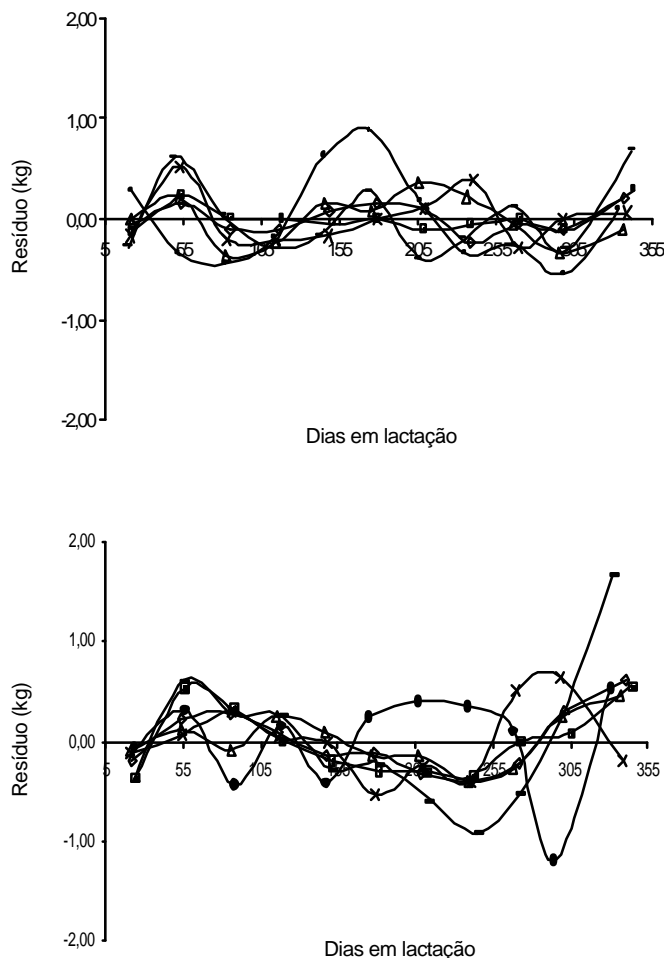

Nível de produção baixo
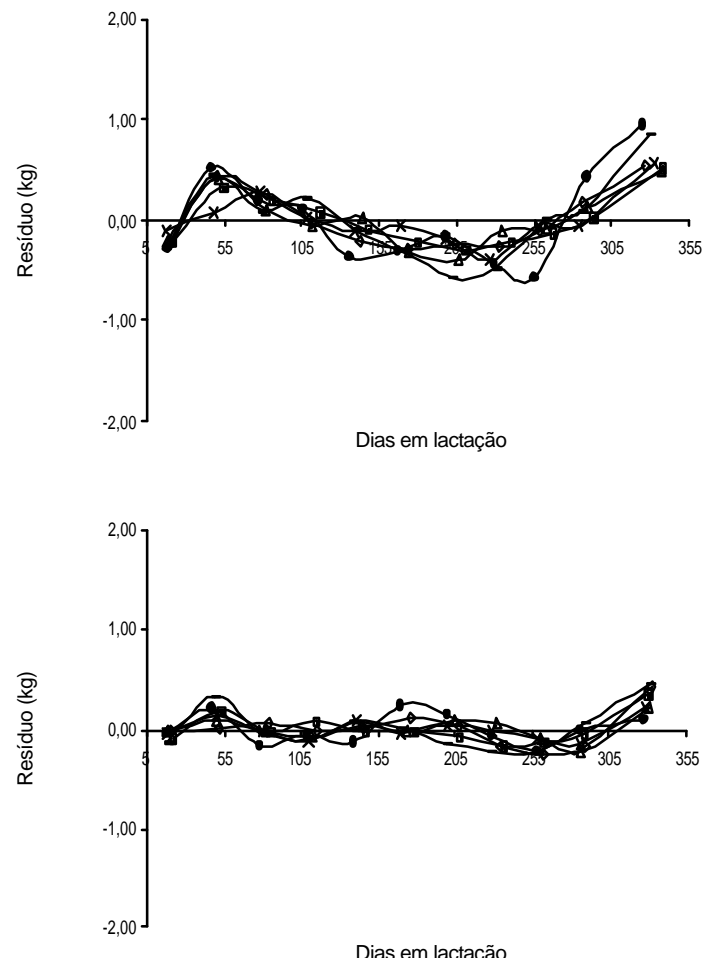

Fiigura 1 - Dispersão dos resíduos para as curvas de lactação de vacas da raça Gir, pertencentes a rebanhos com nível de produção alto e baixo, nas estações de parição seca (Esquerda) e das águas (Direita) nas ordens de parição $(O P=1, \square ; O P=2, \diamond$; $\mathrm{OP}=3, \Delta ; \mathrm{OP}=4, \mathbf{x} ; \mathrm{OP}=5$, - e $\mathrm{OP}>5, \bullet)$.

Estação de parição seca

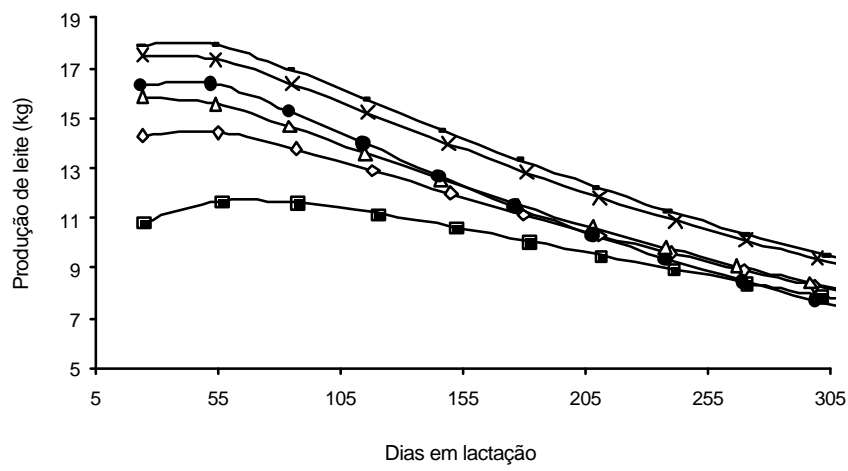

Estação de parição chuvosa

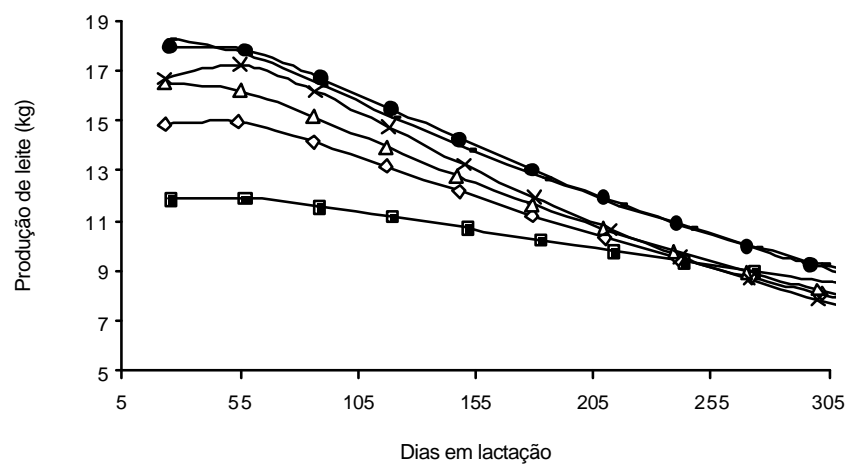

Figura 2 - Curvas de lactação de vacas da raça Gir pertencentes a rebanhos com nível de produção alto nas estações de parição seca e da águas nas ordens de parição $(\mathrm{OP}=1, \boldsymbol{\square} ; \mathrm{OP}=2, \diamond ; \mathrm{OP}=3, \Delta ; \mathrm{OP}=4, \mathbf{x} ; \mathrm{OP}=5$, — e $\mathrm{OP}>5, \bullet)$.

que, quanto menor o valor estimado do parâmetro d, maior o potencial produtivo do animal $\left(\mathrm{Y}_{305}\right)$. Para vacas de nível de produção baixo, independentemente da estação de parição, os valores estimados do parâmetro d forammaiores que para as vacas de nível de produção alto. À medida que aumenta a ordem de parição dos animais, o parâmetro d diminui (Tabela 1), independentemente do nível de produção e da estação de parição. Conseqüentemente, ocorre aumento da produção, o que comprova o relacionamento do parâmetro d com a produção de leite do animal.

$\mathrm{O}$ parâmetro $\mathrm{n}$ também pode ser relacionado a $\mathrm{Y}_{305} \mathrm{e}$, ao contrário do valor do parâmetro d, quanto maior $\mathrm{Y}_{305}$, 
Tabela 2 - Idade média ao parto (IDPM), produção de leite estimada aos 305 dias de lactação $\left(\mathrm{Y}_{305}\right)$, dias até o último controle leiteiro observado (UC), produção de leite total acumulada (P. Ac.), tempo de ocorrência do pico da lactação ( $T_{m}$ ) e produção de leite no dia do pico $\left(\mathrm{Y}_{\mathrm{m}}\right.$.) em vacas da raça Gir provenientes de rebanhos com níveis de produção alto e baixo paridas na estação seca e das águas com ordens de parição $(\mathrm{OP})$ de 1 a 5 e maiores que $5(>5)$

\begin{tabular}{|c|c|c|c|c|c|c|}
\hline OP & IDPM (meses) & $\mathrm{Y}_{305}$ & UC (dias) & P. Ac. (kg) & $\mathrm{T}_{\mathrm{m}}(\mathrm{dias})$ & $\mathrm{Y}_{\mathrm{m}}(\mathrm{kg})$ \\
\hline & \multicolumn{6}{|c|}{ Nível de produção alto } \\
\hline & \multicolumn{6}{|c|}{ Estação seca } \\
\hline 1 & 43 & $3.085,05$ & 345 & $3.385,37$ & 66 & 11,71 \\
\hline 2 & 60 & $3.566,79$ & 338 & $3.825,84$ & 40 & 14,53 \\
\hline 3 & 77 & $3.753,58$ & 337 & $4.007,14$ & 31 & 15,90 \\
\hline 4 & 92 & $4.194,67$ & 340 & $4.504,66$ & 34 & 17,64 \\
\hline 5 & 108 & $4.319,31$ & 343 & $4.662,54$ & 35 & 18,17 \\
\hline$>5$ & 131 & $3.760,98$ & 338 & $3.999,09$ & 36 & 16,60 \\
\hline
\end{tabular}

\begin{tabular}{|c|c|c|c|c|c|c|}
\hline 1 & 43 & $3.212,76$ & 345 & $3.544,56$ & 40 & 11,99 \\
\hline 2 & 59 & $3.622,09$ & 342 & $3.905,57$ & 38 & 15,08 \\
\hline 3 & 76 & $3.837,48$ & 338 & $4.094,91$ & 32 & 16,57 \\
\hline 4 & 94 & $3.928,80$ & 340 & $4.184,78$ & 44 & 17,34 \\
\hline 5 & 109 & $4.246,53$ & 334 & $4.503,55$ & 28 & 18,28 \\
\hline \multirow[t]{3}{*}{$>5$} & 131 & $4.249,28$ & 340 & $4.551,65$ & 37 & 18,13 \\
\hline & \multicolumn{6}{|c|}{ Nível de produção baixo } \\
\hline & \multicolumn{6}{|c|}{ Estação seca } \\
\hline 1 & 47 & $2.195,95$ & 329 & $4.231,45$ & 14 & 8,57 \\
\hline 2 & 63 & $2.691,32$ & 332 & $2.858,48$ & 12 & 11,41 \\
\hline 3 & 80 & $2.875,69$ & 331 & $3.038,61$ & 12 & 12,71 \\
\hline 4 & 95 & $2.880,80$ & 330 & $3.033,39$ & 16 & 12,78 \\
\hline 5 & 111 & $2.877,84$ & 333 & $3.051,46$ & 12 & 12,80 \\
\hline \multirow[t]{2}{*}{$>5$} & 139 & $2.836,25$ & 327 & $2.963,12$ & 11 & 13,24 \\
\hline & \multicolumn{6}{|c|}{ Estação das águas } \\
\hline 1 & 46 & $2.182,85$ & 338 & $2.368,65$ & 1 & 8,66 \\
\hline 2 & 64 & $2.720,36$ & 327 & $2.856,57$ & 1 & 11,96 \\
\hline 3 & 80 & $2.882,49$ & 338 & $3.090,87$ & 1 & 12,89 \\
\hline 4 & 95 & $2.978,51$ & 334 & $3.159,21$ & 1 & 13,80 \\
\hline 5 & 110 & $3.046,61$ & 331 & $3.213,69$ & 1 & 13,94 \\
\hline$>5$ & 141 & $2.802,37$ & 333 & $2.952,04$ & 1 & 13,72 \\
\hline
\end{tabular}

Estação de parição seca

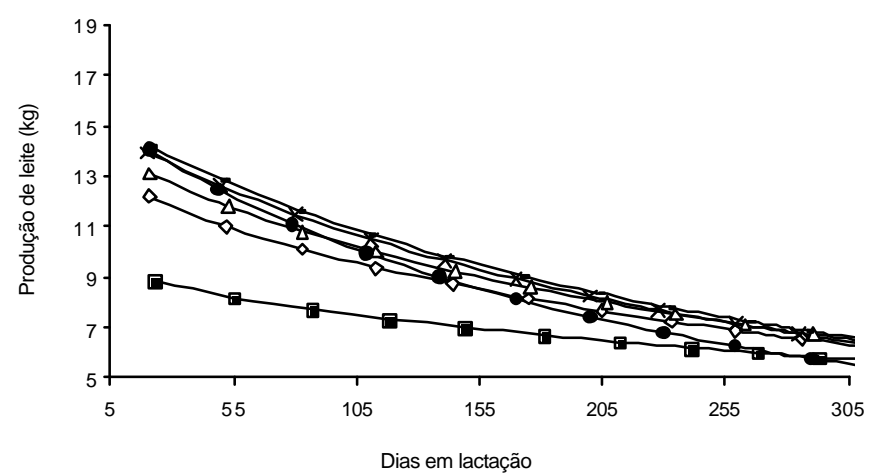

Estação de parição chuvosa

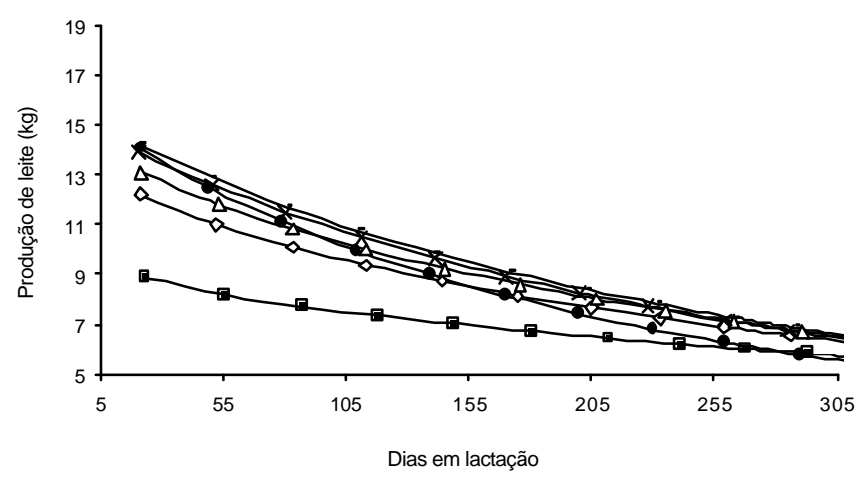

Figura 3 - Curvas baixo, nas estações de lactação de vacas da raça Gir pertencentes a rebanhos com nível de produção de parição seca e da águas nas ordens de parição $(\mathrm{OP}=1, \square ; \mathrm{OP}=2, \diamond ; \mathrm{OP}=3, \Delta ; \mathrm{OP}=4, \mathbf{x} ; \mathrm{OP}=5,-$; e $\mathrm{OP}>5, \bullet)$. 

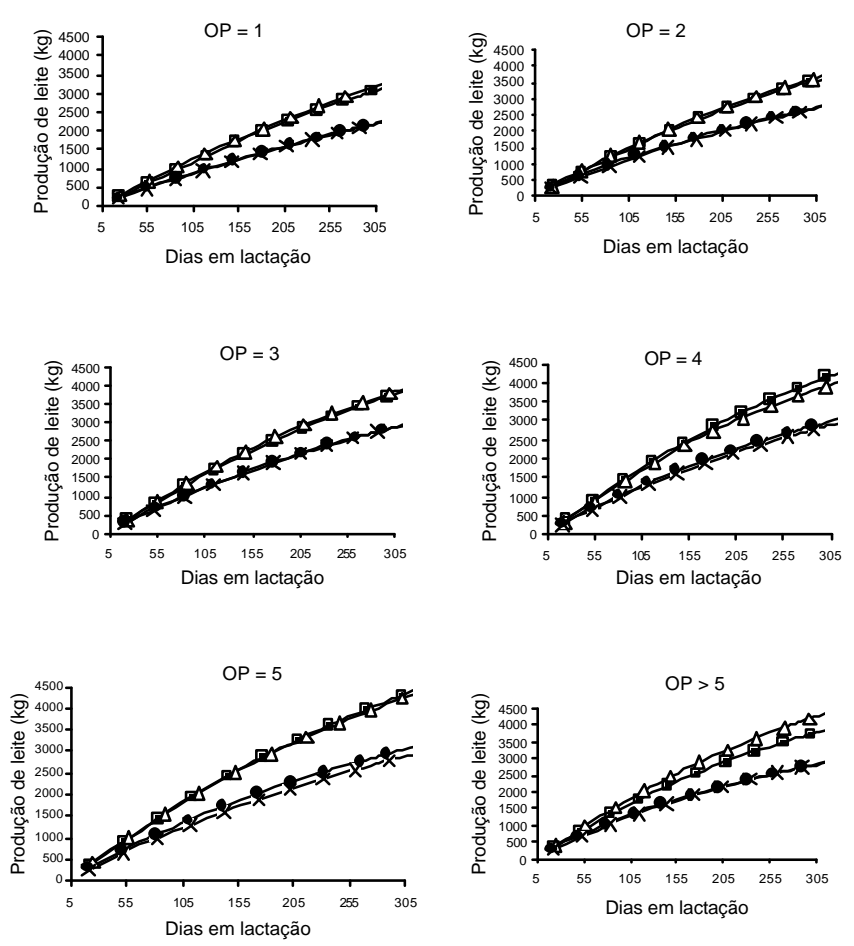

Produção alto com parto na seca; x Produção baixo com parto na seca;

Produção alto com parto nas águas: Produção baixo com parto nas águas.

Figura 4 - Curva de lactação acumulada de vacas da raça Gir pertencentes a rebanhos com nível de produção alto (Prod Alto) e nível de produção baixo (Prod Baixo) nas estações de parição seca (Parto Seca) e da águas (Parto Águas) nas ordens de parição (OP $=1$ a 5 e $>5$ ).

maior o valor correspondente do parâmetro n (Tabela 1). Para vacas de nível de produção alto, os valores do parâmetro n são maiores que os de n para vacas de nível de produção baixo (Tabela 1).

Para encontrar um ponto a partir do qual é possível estimar a produção de leite em 305 dias com menor variabilidade da estimativa, a produção em 305 dias foi estimada a partir das informações obtidas nos diferentes controles leiteiros. A estimativa da produção em 305 dias, independentemente do nível de produção, da estação de parição e da ordem de parição, sofre menor variação a partir de 100 dias de lactação, uma vez que a quantidade de informações necessárias para realização da estimação da produção em 305 dias de lactação é insuficiente e a partir de 100 dias da lactação, a quantidade de informações é suficiente para obter estimativas confiáveis da produção em 305 dias, que podem ser usadas para avaliar o animal em uma fase mais precoce da lactação.
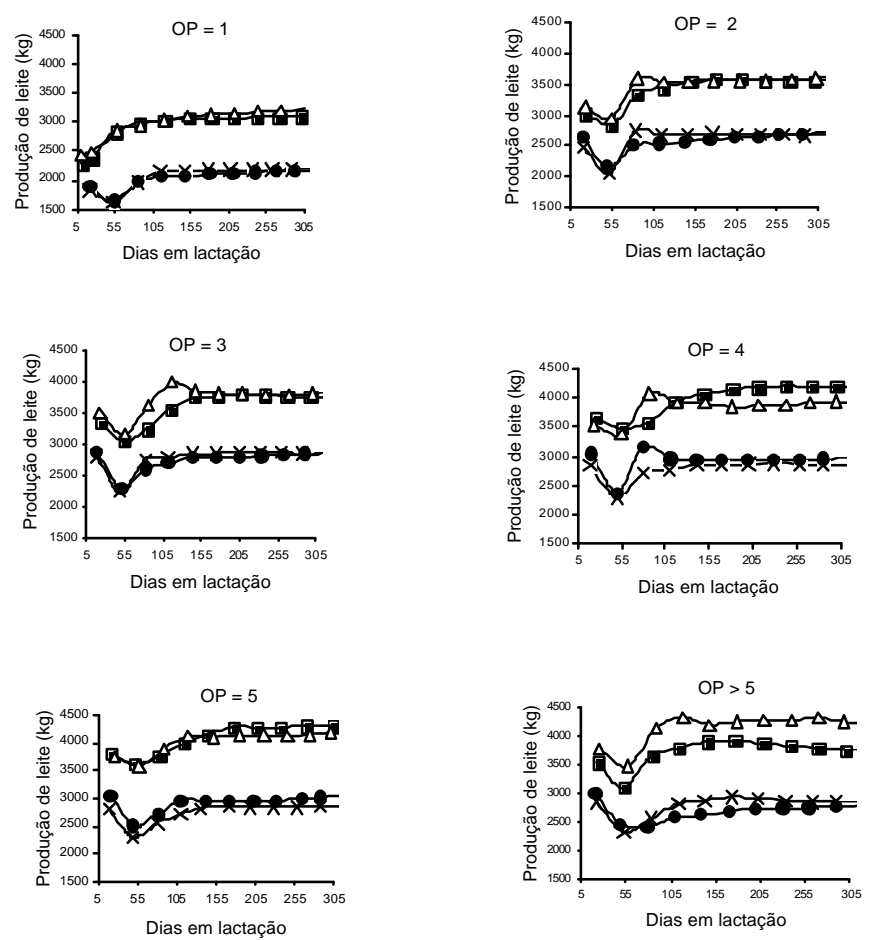

$\square$ Produção alto com parto na seca; $\Delta$ Produção alto com parto nas águas; x Produção baixo com parto na seca; Produção baixo com parto nas águas.

Figura 5 - Estimativa das produções de leite em 305 dias de lactação de vacas da raça Gir, pertencentes a rebanhos com nível de produção alto (Prod Alto) e nível de produção baixo (Prod Baixo) nas estações de parição seca (Parto Seca) e das águas (Parto Águas) nas ordens de parição $(\mathrm{OP}=1$ a 5 e $>5)$.

\section{Conclusões}

A reparametrização da função de Michaelis-Menten constitui alternativa para se obter novas funções para estudos de curvas de lactação diária e acumulada. As novas funções são caracterizadas pela flexibilidade, simplicidade no procedimento de ajuste e pela boa interpretabilidade biológica dos parâmetros. As novas funções utilizadas apresentam-se como funções alternativas para projetar lactações não encerradas, no intuito de serem utilizadas na avaliação genética de animais em programas de melhoramento genético.

\section{Literatura Citada}

KEOWN, J.F.; Van VLECK, L.D. Extending lactation records in progress to 305-day equivalent. Journal of Dairy Science, v.56, n.8, p.1070-1079, 1972. 
KOOPS, W.J.; GROSSMAN, M.; MICHALSKA, E. Multiphasic growth curve analysis in mice. Growth, v.51, p.372-382, 1987.

MORANT, S.V.; GNANASAKTHY, A.A. New approach to the mathematical formulation of lactation curves. Animal Production, v.49, n.2, p.151-162, 1989.

NEAL, H.D.; THORNLEY, J.H.M. The lactation curve in cattle; a mathematical model of the mammary gland. Journal of Agricultura Science, v.101, p.389-400, 1983.

PAPAJCSIK, I.A.; BODERO, J. Modelling lactations curves of Friesian cows in a subtropical climate. Animal Production, v.47, n.1, p.201-207, 1988.

PINEDA, A.M.G. Mathematical functions applied to dairy cow lactation curves. Florida: University of Florida, 1987. 246p.
Thesis (Master of Science) - University of Florida, 1987. STATISTICALANALYSES SYSTEMS - SAS.User's guide for Windows : statistic, Versão 8.0. Cary: SAS Institute, 1998. (CD-ROM).

SCHAEFFER, L.R.; DEKKERS, J.C.M. Random regressions in animal models for test-day production in dairy cattle. In: WORLD CONGRESS ON GENETICS APPLIED TO LIVESTOCK PRODUCTION, 5., 1994, Guelph. Proceedings... Guelph: 1994. v.17, p.443-446.

WILMINK, J.B.M. Adjustment of test-day milk, fat and protein yields fro age, season and stage of lactation. Livestock Production Science, v.16, n.4, p.335-348, 1987.

WOOD, P.D.P. Algebraic model of the lactation curve in cattle. Nature, v.216, n.5111, p.164-165, 1967 